\title{
Índice de refração do fluido ocular como método de avaliação de frescor em peixes de água doce
}

\section{Refractive index of eye fluid as freshness evaluation method of fresh water fishes}

Ana Paula Martins de Souza, ${ }^{*}$ Nelson Nebel Santos, ${ }^{* *}$ Marcelo Sardenberg Teixeira, ${ }^{*}$ Veruschka Paraguassu Romano, ${ }^{* * *}$ Sérgio Carmona de São Clemente, ${ }^{* \star \star *}$ Mônica Queiroz de Freitas ${ }^{* \star * *}$

\begin{abstract}
Resumo
O índice de refração do fluido ocular de três espécies de peixes de água doce foi pesquisado e correlacionado a outras duas análises para determinação de frescor: sensorial e bases voláteis totais. Espécimes de carpa comum (Cyprinus carpio), tambacu (Colossoma macropomum X Piaractus mesopotamicus) e tilápia do Nilo (Oreochromis niloticus), foram armazenados à temperatura entre 0 e $2^{\circ} \mathrm{C}$ por 13, 27 e 21 dias, respectivamente. A carpa, o tambacu e a tilápia se apresentaram impróprios para o consumo, respectivamente, a partir do $13^{\circ}, 7^{\circ}$ e $21^{\circ}$ dias de estocagem, segundo os valores do índice de refração. Nas três espécies mantidas entre $0-2^{\circ} \mathrm{C}$, houve correlação entre o índice de refração e os demais índices de frescor: análise sensorial e o conteúdo de bases voláteis totais. Dessa forma, o índice de refração foi considerado um bom indicador de frescor, devendo, entretanto, ser utilizado em conjunto com outros métodos, para se estabelecer o estado de frescor do pescado e os padrões para cada espécie.
\end{abstract}

Palavras-chave: índice de refração, fluido ocular, humor aquoso, frescor, peixes de água doce, carpa comum, tambacu, tilápia do Nilo, bases voláteis totais, análise sensorial.

\begin{abstract}
The refractive index $(\mathrm{RI})$ of the eye fluid from three fresh water fish species was measured at different storage times and compared to other two indicators of freshness: sensory analysis and total volatile basic amines (TVB). Specimens of common carp (Cyprinus carpio), tambacu (Colossoma macropomum X Piaractus mesopotamicus) and Nilo tilapia (Oreochromis niloticus), were stored at 0 to $2^{\circ} \mathrm{C}$ for 13,27 and 21 days, respectively. Based on the RI values, the commom carp, tambacu and Nilo tilapia were rejected for consumption after 13, 7 and 21 days of storage, respectively. The RI values from the three groups maintained at 0 to $2^{\circ} \mathrm{C}$ showed correlation with TVB and sensory analysis. It can be concluded that RI can be used (along with other indexes) on the freshness evaluation of fresh water fish.
\end{abstract}

Keywords: refractive index, eye fluid, freshness, fresh water fish, total volatile basic amines, TVB, sensory analysis, common carp, tambacu, Nilo tilapia.

\section{Introdução}

Diversos métodos têm sido empregados para demonstrar a qualidade do pescado, dentre os quais se destacam determinação de bases voláteis totais, a análise instrumental de cor, a mensuração de $\mathrm{pH}$, a análise sensorial, a avaliação da textura, a espectroscopia e o índice de refração do fluido ocular.

Devido à rapidez no julgamento da matéria-prima e do produto acabado, como também pela facilidade de execução, a avaliação sensorial é um dos parâmetros utilizados na indústria de pescado para avaliação de qualidade. Por ser um fator determinante na aceitação do consumidor pelo produto, o exame sensorial tem papel fundamental em qualquer programa de controle de qualidade de alimentos, sendo, normalmente, o primeiro teste pelo qual passa o pescado e os demais produtos alimentícios nos órgãos oficiais de controle de qualidade ligados à área de Saúde Pública (Tavares et al., 1998). A FAO, desde 1956, preconiza a avaliação sensorial do pescado como uma ferramenta na avaliação da qualidade do peixe fresco (Reguly et al., 1971).

Conforme aumenta o período post-mortem, os sinais de deterioração se evidenciam, dentre outras características, a partir do turvamento dos líquidos oculares e cristalino, como também pelos bordos irregulares da pupila, antes nitidamente limitados, e pela forma do olho, que se aplaina e se torna côncavo. O humor aquoso se espessa, passando a exibir, ante um raio de luz que a atravesse, um índice de refração distinto do existente quando o animal estava vivo (Ludorff e Meyer, 1978).

A perda de água e aumento na permeabilidade da membrana resulta na redução da pressão intraocular dos olhos causando opacidade e/ou mudanças nas propriedades de transmissão de luz (Yapar e Yetim, 1998).

* Discente Programa de Pós-graduação em Higiene Veterinária e Processamento Tecnológico de Produtos de Origem Animal - Faculdade de Medicina Veterinária - UFF - Niterói - RJ. E-mail para correspondência: ana.pms@uol.com.br.

** Docente da Universidade Federal do Rio Grande do Norte.

*** Médica-veterinária. Instituto Municipal de Medicina Veterinária Jorge Vaitsman - Rio de Janeiro.

**** Docentes Departamento de Tecnologia de Alimentos - Faculdade de Medicina Veterinária - UFF - Niterói, RJ. 
Proctor et al. (1959) e Deufel (1964) em seus estudos consideraram a determinação do índice de refração de fluido dos olhos como um teste útil, tendo o último mencionado sua importância na indicação de frescor em peixes de água doce.

Segundo Wegner (1962), quando o índice de refração atinge o valor de 1,3388 pode-se considerar o peixe como deteriorado. Kietzman et al. (1974) avaliaram o grau de frescor pela determinação do índice de refração do humor aquoso em bacalhau (Gadus morrhua), bacalhau saithe (Pollachius virens), arinca (Melanogrammus aeglefinus), merluza (Merluccius merluccius) e bacalhau ling (Molva molva) e estabeleceram a relação entre o grau de frescor e o valor do índice de refração do fluido ocular (IR) de algumas espécies. Afirmaram, ainda, que até 1,3355 de valor de IR do fluido ocular indica um peixe muito fresco, enquanto o valor de 1,3390 se refere à deterioração para a maioria das espécies de peixes. Nagashima et al. (1990) também utilizaram algumas propriedades do fluido ocular para a avaliação do frescor de pescado através do índice de refração. Queiroz et al. (1993) concluíram que o índice de refração do fluido ocular, bases voláteis totais e pH apresentaram alto coeficiente de correlação com a avaliação sensorial.

Considerando o fato da determinação do frescor de peixes através do índice de refração do fluido ocular requerer estudos específicos para cada espécie de peixe, aliado à importância crescente do pescado cultivado em água doce e da necessidade de técnicas rápidas, baratas e efetivas para a determinação do frescor dessas espécies, este trabalho objetivou: i) estudar o comportamento do índice de refração, das bases voláteis totais e das características sensoriais, em peixes de água doce (carpa comum, tambacu e tilápia do Nilo) estocados à temperatura de $0-2^{\circ} \mathrm{C}$; ii) testar correlações entre os parâmetros empregados como indicativos de frescor nos espécimes em estudo; iii) determinar o prazo de vida comercial dos espécimes em estudo; e iv) observar a eficiência do Refratômetro Mettler Toledo RE 50 na determinação do índice de refração do fluido ocular.

\section{Material e métodos}

Foram capturados 35 exemplares de carpa comum (Cyprinus carpio), 25 de tambacu, híbrido originado do tambaqui (Colossoma macropomum) e do pacu (Piaractus mesopotamicus) e 35 de tilápia do Nilo (Oreochromis niloticus) de um criatório de peixes de água doce localizado no município de Magé/RJ.

Os peixes foram obtidos em uma única captura e cada espécie foi dividida em grupos de cinco exemplares, embalados individualmente em sacos de polietileno e armazenados em caixas isotérmicas, cobertos em seguida com gelo, de forma a manter a temperatura entre 0 e $2^{\circ} \mathrm{C}$. Os peixes foram transportados por um período não superior a duas horas, uma parte dos peixes foi analisada e outra estocada sob as mesmas condições para estudo posterior. Cada grupo foi analisado química, instrumental e sensorialmente, em intervalos específicos: as carpas comuns foram analisadas até 13 dias (dia zero, dia 3 , dia 5 , dia 7 , dia 10, dia 12, dia 13), as tilápias do Nilo foram analisadas até 21 dias (dias 0, 7, 13, 21) e os tambacus até 27 dias (dias $0,7,13,21,27$ ).

\section{Avaliação sensorial}

A avaliação sensorial constou de análise do pescado no estado cru. $O$ frescor do peixe in natura foi avaliado através do quadro de análise sensorial criado por Gelman et al. (1989) para a avaliação de carpa comum, sendo adaptado para a avaliação das outras duas espécies (tambacu e da tilápia do Nilo). Na análise sensorial foram avaliados a aparência geral, cor, odor e firmeza da carne, guelras, olhos, vísceras e superfície do corpo. Todos os parâmetros foram colocados em escala decrescente de 10 a 0 , em que o valor 10 representava o maior frescor do pescado.

A avaliação sensorial foi conduzida sob condições laboratoriais, sobre uma bancada de mármore de cor clara, utilizando luz branca. Os peixes foram desembalados, colocados sobre bandejas de cor clara e analisados individualmente. Procedeu-se à pontuação das diversas características e, através de média aritmética, obteve-se o grau de qualidade médio em que o peixe se encontrava.

\section{Determinação de umidade}

A determinação do teor de umidade foi realizada pelo método de estufa a $105^{\circ} \mathrm{C}$ de acordo com o método adotado pelo LANARA (Brasil, 1981).

\section{Determinação de bases voláteis totais}

O teor de bases voláteis totais foi determinado através do método de microdifusão de acordo com o método adotado pelo LANARA (Brasil, 1981).

\section{Determinação do índice de refração}

Para a determinação do índice de refração foi utilizado o Refratômetro Mettler Toledo RE 50 seguindo-se as instruções do fabricante para a calibração e mensuração.

O humor aquoso foi extraído através da introdução de uma agulha na região esclerocorneal em direção ao centro do bulbo. A dimensão dessas agulhas variou de acordo com 0 período de estocagem: $20 \mathrm{~mm} \times 5,5 \mathrm{~mm}$ no período inicial, $25 \mathrm{~mm} \times 7 \mathrm{~mm}$ no período intermediário e $30 \mathrm{~mm} \times 8 \mathrm{~mm}$ no final de armazenamento. Esse aumento no calibre das agulhas se fez necessário porque conforme aumentava o período de estocagem, a retirada do humor aquoso dos olhos dos peixes ficava dificultada pela infiltração de sangue e material particulado no fluido ocular.

Foram obtidas conjuntamente amostras dos dois olhos, sendo o volume extraído em média de 0,4 ml, variando em função da espécie e do tamanho do peixe analisado. Após a calibração do aparelho com água destilada à temperatura de $20^{\circ} \mathrm{C}$, era feita a determinação do índice de refração do humor aquoso. Tal determinação durava aproximadamente 10 segundos.

\section{Análise estatística}

Para o estudo estatístico foi utilizado o Programa Estatístico SPSS versão 8.0, utilizando-se análises estatísticas descritivas sobre as variáveis, com o uso de testes de hipótese de Wilcoxon-Mann-Whitney e de Kruskal-Wallis para determinar a existência ou não de diferenças significativas (a um nível de significância de $5 \%$ ) entre os grupos estudados. Foi também realizada uma análise de correlação, com base no coeficiente de correlação de Pearson, entre o índice de refração e os demais índices de frescor no decorrer do período de estocagem. 


\section{Resultados e discussão}

\section{Carpa comum (Cyprinus Carpio)}

Os valores médios de índice de refração do fluido ocular, de bases voláteis totais e da avaliação sensorial e seus respectivos desvios-padrões da carpa comum estocada à temperatura de 0 e $2^{\circ} \mathrm{C}$, em diferentes dias de estocagem, encontram-se na Tabela 1.

Tabela 1: Valor médio e desvio-padrão dos índices de frescor da carpa comum (Cyprinus carpio) mantida entre 0 e $2^{\circ} \mathrm{C}$ durante 13 dias

\begin{tabular}{ccrr}
\hline Estocagem (Dia) & IR & \multicolumn{1}{c}{ BVT } & AS \\
\hline 0 & $1,3351 \pm 0,00011$ & $7,90 \pm 1,56$ & 10,0 \\
3 & $1,3352 \pm 0,00011$ & $12,30 \pm 1,79$ & 9,0 \\
5 & $1,3355 \pm 0,00013$ & $12,94 \pm 1,23$ & 8,5 \\
7 & $1,3364 \pm 0,00069$ & $12,81 \pm 1,95$ & 7,1 \\
10 & $1,3384 \pm 0,00256$ & $14,02 \pm 1,88$ & 6,0 \\
12 & $1,3397 \pm 0,00269$ & $17,66 \pm 5,28$ & 5,8 \\
13 & $1,3432 \pm 0,00360$ & $17,02 \pm 1,62$ & 4,8 \\
\hline
\end{tabular}

$\mathrm{IR}=$ Índice de refração; BVT = Bases voláteis totais $(\mathrm{mg} / 100 \mathrm{~g}) ; \mathrm{AS}=$ Avaliação sensorial

Comparando os dias de estocagens pelo Teste de KruskalWallis, observaram-se diferenças significativas nos correspondentes índices entre a maior parte dos dias. Em relação ao índice de refração não foram encontradas diferenças significativas (pela aplicação do Teste de Wilcoxon-MannWhitney) entre os dias 0 e 3, e entre os dias 10, 12 e 13 (entre si). Quanto às bases voláteis totais, não foram encontradas diferenças significativas entre os dias 3, 5, 7 e 10 (entre si).

De acordo com os padrões estabelecidos por Proctor et al. (1959), para arinca, Wegner (1962) para bacalhaus e Kietzmann et al. (1974) para bacalhau, bacalhau saithe, arinca, merluza e bacalhau ling, o índice de refração do fluido dos olhos das tilápias recém-capturadas esteve dentro dos limites considerados de qualidade excelente ou muito boa. $\mathrm{O}$ mesmo foi observado para a estocagem no dia 3 e 5, mas segundo o mesmo autor, os valores encontrados no dia 5 são considerados em termos de qualidade como boa a regular. Para a carpa comum mantida em gelo no dia 7 , segundo Proctor et al. (1959) e Kietzmann et al. (1974) pode ser considerada como de qualidade regular a boa, já de acordo com Wegner (1962), como de qualidade boa. Em média, os espécimes avaliados nos dias 10 e 12 obtiveram valores que permitem classificá-los como de qualidade regular, segundo Proctor et al. (1959), Wegner (1962) e Kietzmann et al. (1974). Apenas, a partir do dia 13 de estocagem em gelo, os peixes são considerados não comerciáveis (Proctor et al., 1959) ou deteriorados (Wegner, 1962 e Kietzmann et al., 1974).

Além disso, os graus de evolução dos diferentes parâmetros sensoriais não foram os mesmos durante o período estudado. A cor das guelras e a condição das vísceras se alteraram mais lentamente que a forma e a turvação dos olhos, concordando com as observações de Gelman et al. (1989).

Os valores de BVT iniciais apresentaram flutuações mínimas, até um aumento que ocorre no final do período de estocagem quando o peixe estava completamente deteriorado, concordando com o trabalho realizado por Gelman et al. (1989). Segundo os mesmos autores, os resultados do teste de BVT não se mostraram como indicadores apropriados de frescor em carpa comum mantida à temperatura ambiente.

Durante a estocagem da carpa comum, por 13 dias, foi observada correlação entre o índice de refração do fluido ocular e o conteúdo de bases voláteis totais, com coeficiente de correlação (r) de 0,620; e correlação negativa entre o índice de refração e a avaliação sensorial com valor de $r$ de $-0,751$. Devido aos escores da escala estruturada da análise sensorial decrescerem em função da perda de qualidade, também foi observada uma correlação negativa, com valor $r$ de $-0,707$, entre as bases voláteis totais e a avaliação sensorial.

\section{Tambacu (Colossoma Macropomum X Piaractus Mesopotamicus)}

No estudo deste peixe, foram realizadas análises semanais, iniciando-se no dia zero (dia da coleta) e finalizando no dia 27. Os resultados médios e os respectivos desvios-padrões dos parâmetros estudados em função do tempo de estocagem podem ser observados na Tabela 2.

Tabela 2: Média e desvio-padrão dos índices de frescor do tambacu (Colossoma Macropomum $X$ Piaractus Mesopotamicus) mantido entre 0 e $2^{\circ} \mathrm{C}$ durante 27 dias

\begin{tabular}{ccrc}
\hline Estocagem (Dia) & IR & BVT & AS \\
\hline 0 & $1,3353 \pm 0,00013$ & $4,16 \pm 1,30$ & 10,0 \\
7 & $1,3424 \pm 0,00358$ & $13,10 \pm 0,92$ & 7,5 \\
13 & $1,3425 \pm 0,00346$ & $12,84 \pm 0,61$ & 6,0 \\
21 & $1,3460 \pm 0,00279$ & $13,36 \pm 0,63$ & 4,4 \\
27 & $1,3451 \pm 0,00335$ & $14,12 \pm 1,43$ & 3,9 \\
\hline IR = Índice de refração; BVT = Bases voláteis totais (mg/100g); AS = \\
Avaliação sensorial
\end{tabular}

Tanto para o índice de refração quanto para as bases voláteis totais, pela utilização dos testes de Kruskal-Wallis e de Wilcoxon-Mann-Whitney, só foram observadas diferenças significativas $(p<0,05)$ entre o dia 0 e os demais dias $(7,13$, 21 e 27). De acordo com os padrões estabelecidos por Proctor et al. (1959), Wegner (1962), e Kietzmann et al. (1974), o índice de refração do fluido dos olhos dos tambacus recémcapturados esteve dentro dos limites considerados como de qualidade excelente, muito boa ou muito fresca. Para tambacus mantidos em gelo a partir do 7ㅇ dia, a média do índice de refração esteve entre os intervalos considerados como não comercializáveis segundo Proctor et al. (1959) e impróprios para consumo de acordo com Wegner (1962) e Kietzmann et al. (1974). Os resultados obtidos na presente pesquisa estão em desacordo com os autores citados anteriormente, no que diz respeito aos valores da avaliação sensorial, em que o tempo de vida comercial, com escore sensorial até seis, foi observado no dia 13 de estocagem.

No experimento realizado com a carpa estocada à temperatura de 0 e $2^{\circ} \mathrm{C}$, a cor das guelras, a textura, a cor e o odor do muco, entre outras características se alteraram mais lentamente quando comparadas a forma e a turvação dos olhos, concordando com os resultados obtidos por Gelman 
et al. (1989). O presente estudo observou a grande importância da aparência dos olhos na avaliação sensorial do pescado, corroborando aos resultados obtidos por Love (1954) e Queiroz et al. (1993). O conteúdo de bases voláteis totais se correlacionou com o índice de refração do fluido ocular com valor de 0,785. A evolução de ambos os índices foi muito semelhante, pois, como em relação aos valores de índice de refração, a partir do dia 7 de estocagem, os valores de bases voláteis totais não se alteraram significativamente $(p<0,05)$. Ocorreu também uma correlação negativa com valor $r$ de 0,778 , entre o índice de refração e a avaliação sensorial. Foi observada, ainda, uma correlação entre a análise sensorial e o conteúdo de bases voláteis totais, com valor de $r$ de -0,838.

\section{Tilápia do Nilo (Oreochromis Niloticus)}

No estudo da tilápia do Nilo foram realizadas análises semanais, iniciando-se no dia zero (dia da coleta) e finalizando no dia 21. Os valores médios de índice de refração do fluido ocular, de bases voláteis totais e da avaliação sensorial e seus respectivos desvios-padrões encontram-se na Tabela 3.

Tabela 3: Valor médio e desvio-padrão dos índices de frescor da tilápia do Nilo (Oreochromis niloticus) mantida entre 0 e $2^{\circ} \mathrm{C}$ durante 21 dias

\begin{tabular}{cccc}
\hline Estocagem (Dia) & $\mathrm{IR}$ & BVT & AS \\
\hline 0 & $1,3358 \pm 0,00047$ & $5,56 \pm 1,66$ & 10,0 \\
7 & $1,3420 \pm 0,00155$ & $14,70 \pm 0,90$ & 7,0 \\
13 & $1,3424 \pm 0,00203$ & $12,34 \pm 1,52$ & 4,6 \\
21 & $1,3436 \pm 0,00126$ & $12,40 \pm 1,86$ & 4,1 \\
\hline
\end{tabular}

IR = Índice de refração; BVT = Bases voláteis totais $(\mathrm{mg} / 100 \mathrm{~g}) ; A S=$ Avaliação sensorial

O índice de refração se correlacionou com as bases voláteis totais com valor $r$ de 0,667 , e com a análise sensorial, com valor $r$ de $-0,817$. A correlação entre as bases voláteis totais e

\section{Apoio financeiro: $\mathrm{CNPq}$}

\section{Referências}

BRASIL, Ministério da Agricultura. Métodos analíticos oficiais para controle de produtos de origem animal e seus ingredientes, II: métodos físicos e químicos. Brasília, DF, LANARA - Laboratório Nacional de Referência Animal - Secretaria Nacional de Defesa Agropecuária, 1981.

DEUFEL, J. Comparative studies on the determination of the degree of freshness of fresh water fish. Lebensmitte - Junters $u$ - Forsch, Berlim, v. 123, n. 5, p. 354-361, 1964.

GELMAN, A.; PASTEUR, R.; RAVE, M. Quality changes and storage life of common carp (Cyprinus carpio) at various storage temperatures. $J$. Sci. Food Agric., Israel, v. 52, n .2, p. 231-247, 1989.

KIETZMANN, U. et al. Inspección veterinaria de pescados. Espanha: Acribia, 1974. 326 p.

LOVE, R.M. Post-mortem changes in the lenses of fish eyes:assessment of storage time and fish quality. J Sci Food Agric, v. 5, n. 12, dez, 1954.

LUDORFF, W.; MEYER, V. El pescado y los productos de la pesca. Espanha: Acribia, 1978. 342 p.

NAGASHIMA, Y.; TANAKA, M.; TAGUCHI, T. Evaluation of yellowfin tuna freshness by physicochemical properties of the eye fluid. $J$ Tokyo Univ Fish, v. 77, p. 153, 1990. os dados da avaliação sensorial ficou um pouco abaixo das demais com valor de $r$ de 0,577 . De acordo com os padrões estabelecidos por Proctor et al. (1959), Wegner (1962) e Kietzmann et al. (1974), o índice de refração do fluido dos olhos das tilápias recém-capturadas esteve dentro dos limites considerados como de qualidade excelente ou muito boa. Para tilápias mantidas em gelo a partir do 7ㅇ dia, a média esteve entre os intervalos considerados como não comercializáveis segundo Proctor et al. (1959) e deterioradas de acordo com Wegner (1962) e Kietzmann et al. (1974).

Apesar de estatisticamente existir correlação entre os teores de bases voláteis totais e o índice de refração do fluido ocular, os resultados das bases voláteis totais isoladamente não permitem avaliar o grau de frescor da tilápia do Nilo, assim como no trabalho de Gelman et al. (1989) em seu estudo sobre a vida de prateleira da carpa comum.

\section{Conclusão}

Os resultados sugerem que o índice de refração do humor aquoso pode ser utilizado como indicador de frescor dos peixes de água doce: carpa comum (Cyprinus carpio), tambacu (Colossoma macropomum X Piaractus mesopotamicus) e tilápia do Nilo (Oreochromis niloticus), estocados à temperatura entre 0 e $2^{\circ} \mathrm{C}$. Existe semelhança entre o padrão de evolução do índice de refração e a avaliação sensorial durante a determinação da vida comercial das espécies estudadas. O padrão de evolução das bases voláteis totais se mostrou como um indicador apropriado de frescor no tambacu, não tendo sido observado nas demais espécies analisadas.

O Refratômetro Mettler Toledo RE 50, utilizado para a medição do índice de refração do fluido ocular em pescado, mostrouse eficaz, pois além das leituras rápidas (cerca de 10 segundos), os resultados obtiveram boa repetibilidade e precisão, podendo ser utilizado um volume bem pequeno de amostra (cerca de 0,4 ml). Apesar de ser considerado um bom indicador de frescor em pescado, o índice de refração do fluido ocular precisa ser mais estudado, de forma a se estabelecer uma escala de valores próprios para cada espécie de peixe de água doce, o que constitui a verdadeira dificuldade na aplicabilidade deste procedimento.

PROCTOR, B. E. etal. Rapid determination of the quality of whole eviscerated haddock. Food Tech, Champaign, v. 13, n. 4, p. 224-228, 1959.

QUEIROZ, M. I.; TREPTOW, R. O.; QUEIROZ, E. M. Escala sensorial para avaliação do frescor do pescado estocado em gelo. Boletim do Centro de Pesquisa e Processamento de Alimentos, Curitiba, v. 11, n. 2, p. 91-102, jul./dez. 1993.

REGULY, J.C.; MUNSTOCK, A.; KOETZ, P.R. Avaliação qualitativa do pescado marinho de expressão industrial no sul do Brasil. Rio Grande: FURG, 1971.

TAVARES, M. et al. Métodos sensoriais, físicos e químicos para análise de pescado. Controle de qualidade de pescado. São Paulo, Loyola. São Paulo, 1998, p. 117-134.

WEGNER, H. J. Further studies on refractometry as a means of estimating freshness of marine fish. Arch Lebensmittelhyg, Hannover, v. 13, p. 90-91, 1962.

YAPAR, A.; YETIM, H. Determination of anchovy freshness by refractive index of eye fluid. Food Research International, Inglaterra, v. 31, n. 10, p. 693-695, 1998. 